\title{
Sarcoidosis with combined kidney damage - kidney stones, granulomatous interstitial nephritis and acute kidney injury
}

\author{
Elena V Zakharova ${ }^{1 *}$ and Ekaterina S Stolyarevich ${ }^{2}$ \\ ${ }^{1}$ Head of Nephrology Department, Botkin Memorial Hospital, Moscow, Russian Federation \\ ${ }^{2}$ Head of Nephropathology Department, City Nephrology Centre, Moscow, Russian Federation
}

\begin{abstract}
Sarcoidosis is a systemic disease with unknown cause, characterized by noncaseating granulomas, predominantly in the lungs and intrathoracic lymph nodes. Sarcoidosis is associated with a broad spectrum of extrapulmonary involvement, including renal manifestations. Interstitial granulomatous nephritis is the most typical finding; another characteristic feature is kidney damage due to disordered calcium homeostasis, driven by calcitriol hyperactivity of macrophages, and leading to acute kidney injury, nephrocalcinosis, nephrolithiasis, tubular dysfunction etc. Treatment is always required for renal and other end-organ damage; corticosteroids are the mainstay of therapy. We present a case of sarcoidosis with both interstitial granulomas and kidney stones, complicated by acute kidney injury, and successfully treated with corticosteroids.
\end{abstract}

\section{Background}

Sarcoidosis is a systemic disease, affecting predominantly lungs and intrathoracic lymph nodes (75-90\%), but also involving many other organs, including skin, eyes, bone marrow, liver, spleen, nose, throat, salivary glands, joints, central nervous system, heart, endocrine and gastrointestinal system, and also kidneys $[1,2]$.

The cause of the disease is not known - as yet, no bacterial, fungal, or viral antigen has been consistently isolated from the sarcoidosis lesions. Sarcoidosis is neither a malignant nor an autoimmune disease, however, both genetic and environmental factors seem to play a role, and reaction to an unidentified antigen is suspected, despite the cellular immune response occurs in the absence of a clearly defined immunological target [3-6].

The characteristic presentation of sarcoidosis is noncaseating granulomas, predominantly in the lungs and intrathoracic lymph nodes, but also in kidney interstitium. However sarcoidosis is associated with a broad spectrum of renal manifestations beyond granulomatous interstitial nephritis. The incidence of renal involvement ranges from $23 \%$ up to $48 \%$ with a wide spectrum of abnormalities $[1,2,7,8]$.

Interstitial granulomatous nephritis is the most typical histological finding, but development of renal insufficiency is unusual, while the most important cause of renal dysfunction is a disordered calcium metabolism. Hypercalcaemia, caused by calcitriol hyperactivity of macrophages, presents in $10-17 \%$ of patients with sarcoidosis, hypercalciuria in $40-62 \%$ of patients [8-10]. A disordered calcium homeostasis is responsible for the development of renal dysfunction by several different mechanisms. Hypercalcaemia promotes a decrease in glomerular filtration rate by vasoconstriction of the afferent arteriole; inhibits sodium-potassium ATP-ase leading to urinary sodium wasting with polyuria and dehydration; urinary concentration is impaired by a decreased sensitivity to anti-diuretic hormone; acute tubular necrosis may result from intracellular calcium overload and tubular obstruction by calcium precipitates. Hypercalciuria predisposes to nephrolithiasis and obstructive uropathy. Untreated, chronic hypercalcaemia and hypercalciuria cause a progressive tubulointerstitial inflammation with associated calcium deposits leading to nephrocalcinosis, which is the leading cause of chronic kidney disease in sarcoidosis. In the acute phase, the consequences of hypercalcaemia and hypercalciuria are reversible. Once fibrotic lesions have developed due to longstanding disease, the damage is irreversible $[9,11,12]$.

Tubular dysfunction is frequently associated both with hypercalcaemia and granulomatous interstitial nephritis. It may present as isolated proximal or distal tubular acidosis, Fanconi syndrome, urinary concentration defects or metabolic alkalosis. Obstructive uropathy is usually due to nephrolithiasis. Retroperitoneal fibrosis, retroperitoneal lymph nodes and ureteral, urethral or bladder obstruction by sarcoid involvement can also cause obstructive disease $[9,13]$.

Granulomatous angiitis is a rare complication of sarcoidosis and can involve the renal artery; it is often accompanied by arterial hypertension [12]. Glomerular involvement in sarcoidosis is rare, although a variety of different lesions have been described including membranous nephropathy, focal segmental sclerosis, IgA nephropathy and crescentic glomerulonephritis [14,15]. Different types of renal sarcoidosis had also been reported to coexist $[7,11,12]$.

Treatment is always required for renal, cardiac, ocular and neurologic manifestations of sarcoidosis, given the substantial risk

Correspondence to: Elena V Zakharova, Nephrology Department, Head, Botkin Memorial Hospita 125284, 2-nd Botkinsky proezd, 5, Moscow, Russian Federation, Tel: +7 967134 6936; Fax: +7 495945 1756, E-mail: helena.zakharova@gmail.com com

Key words: sarcoidosis, granulomas, interstitial nephritis, hypercalcaemia, kidney stones, corticosteroids

Received: March 19, 2016; Accepted: April 15, 2016; Published: April 18, 2016 
of end-organ damage [16-18]. Patients with severe $(>3.5 \mathrm{mmol} / \mathrm{L})$ or symptomatic hypercalcaemia require intravenous saline hydration as initial therapy [19]. The specific treatment is aimed to treatment of the underlying disorder. Corticosteroids are the mainstay of therapy. Most authors recommend starting with an initial dose of $0.5-1 \mathrm{mg}$ per $\mathrm{kg}$ oral prednisone once daily depending on the severity of the disease. After 4 weeks of treatment, the dose can be tapered by $5 \mathrm{mg}$ each week until a daily dose of 5-10 mg is reached. Noncorticosteroid agents are being increasingly tried. Common indications for the initiation of such agents include steroid-resistant disease, intolerable adverse effects, or patient desire not to take corticosteroids [7,9-12,16,17].

Here we present a case of sarcoidosis with combined kidney involvement, successfully treated with corticosteroids.

\section{Case presentation}

Asian male, 39 years old, was referred to Nephrology, Moscow Botkin Memorial Hospital, September 2015.

Main complains: general weakness, decreased appetite, weight loss, frequent urination and eye redness.

Previous medical history: combined hemorrhoid since age of 20 , pneumonia 5 years ago.

History of current disease: February 2014 he developed unexplained painful erythematous rush on lower extremities, resolved spontaneously. March 2014 he underwent surgery due to hemorrhoids bleeding. Since that time erythematous rush recurred, over next year patient experienced chest pain, headache, low-grade fever, weight loss, constipation, dry mouth, and noticed hoarseness. Outpatient workup in December 2014 found elevated serum creatinine, no further investigation was performed.

August 2015 he was admitted to local urology clinic with his serum creatinine raised up to $377 \mu \mathrm{mol} / \mathrm{L}$, kidney ultrasound and plain urography revealed non-obstructive stone in the left pelvis. He underwent percutaneous nephrolithotomy and simultaneous left kidney biopsy. Biopsy specimens were sent for evaluation to Nephropathology Department, Moscow City Nephrology Centre.

Kidney biopsy: Pathology evaluation by light microscopy with H\&E, PAS and Masson-trichrome staining's (Figures 1-4) showed 16 otherwise normal glomeruli. Interstitial changes were represented by diffuse-focal lymphohystiocytic infiltration and multiple large granulomas, constituted by epithelioid and giant multinucleated cells, without significant periphery lymphoid reaction. Severe interstitial oedema and diffuse-focal interstitial fibrosis and tubular atrophy were also seen. Arteries and arterioles were otherwise normal. Immune staining's were negative for $\operatorname{Ig} A, \operatorname{IgG}, \operatorname{IgM}, \mathrm{C} 3$ and $\kappa$ and $\lambda$ light chains and fibrinogen. Pathology conclusion: granulomatous interstitial nephritis.

Patient was referred to our unit for consultation.

Urgent work-up and treatment: point-of-care blood test for calcium was performed and showed total calcium $3.39 \mathrm{mmol} / \mathrm{L}$. Patient was urgently admitted, and started with normal saline infusions 2500 $\mathrm{ml} /$ day.

At admission: conscious, alert, oriented, slightly depressed. Body temperature $37.4^{\circ} \mathrm{C}, \mathrm{RR} 18$ per minute, pulse regular 80 per minute, BP 130/80 mm Hg. Pale, undernourished. Dry skin, no peripheral oedema, neither palpable peripheral lymph nodes. Joints: no swelling,

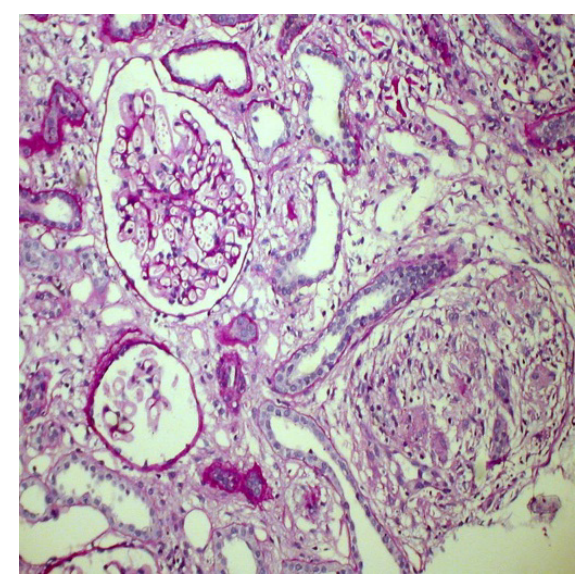

Figure 1. Noncaseating granuloma and otherwise normal glomerulus. PAS x 200.

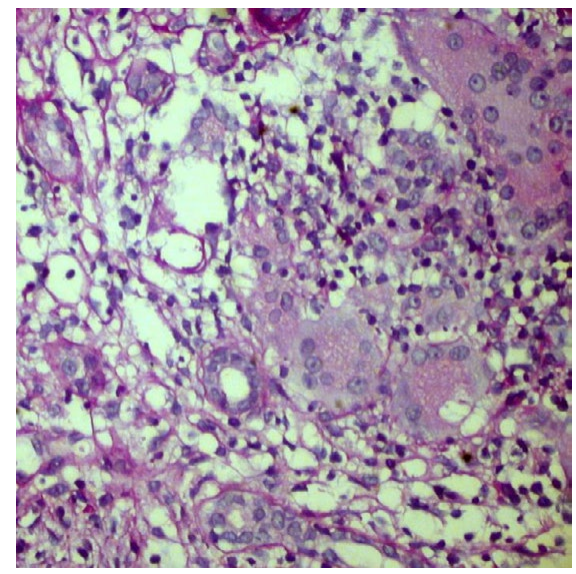

Figure 2. Giant multinuclear cells in the interstitium. PAS x 400 .

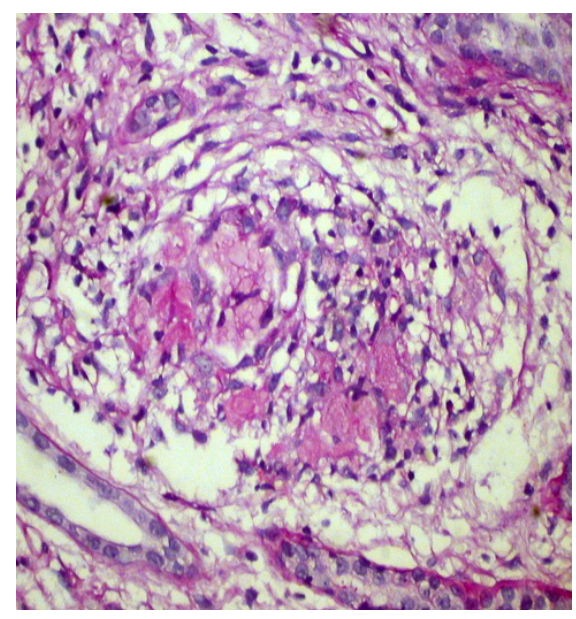

Figure 3. Interstitial noncaseating granuloma. H\&E x 400.

movements not restricted. Eyes: moderate ocular injection. Hoarseness, ENT and neck otherwise normal. Lungs: no dullness to percussion, any rhonchi, wheezes or rubs. Heart: regular rhythm, no murmur. Abdomen soft, non-tender, bowel sounds normal. Liver $+1 \mathrm{~cm}$ below rib arch, non-painful, spleen and kidneys not felt. Frequent urination, mainly nocturnal. Urine is colourless, urine output $3200 \mathrm{ml} /$ day. 


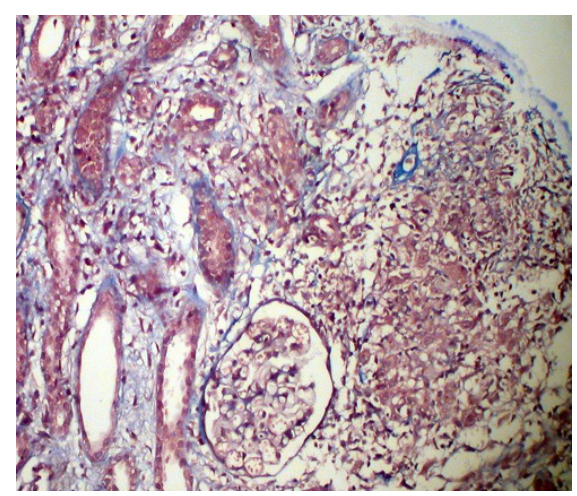

Figure 4. Interstitial noncaseating granuloma, tubular atrophy and otherwise normal glomeruli. Masson x 200 .

\section{Work up:}

Total blood count: $\mathrm{Hb} 11.4 \mathrm{~g} / \mathrm{L}$, WBC $7.8 \times 10^{9} / \mathrm{L}$ (eosinophil's $5.8 \%)$, Plt $417 \times 10^{9} / \mathrm{L}$, ESR $36 \mathrm{~mm} / \mathrm{h}$

Blood chemistry: creatinine $249 \mu \mathrm{mol} / \mathrm{L}$, urea $10.2 \mathrm{mmol} / \mathrm{L}$, total protein $61 \mathrm{~g} / \mathrm{L}$, albumin $31 \mathrm{~g} / \mathrm{L}$, potassium $3.3 \mathrm{mmol} / \mathrm{L}$, ionized calcium $1.63 \mathrm{mmol} / \mathrm{L}, \mathrm{pH}$ 7.27. Phosphorous, serum iron, sodium, chloride, uric acid, glucose, albumin, cholesterol, bilirubin, liver enzymes within normal range.

Urinalysis: SG 1008, $\mathrm{pH} 7$, protein $0.37 \mathrm{~g} / \mathrm{L}$, glucose $2.6 \mathrm{mmol} / \mathrm{L}$, RBC 1-3, WBC 40-45 hpf.

\section{Urine chemistry: not tested}

Urine culture: negative.

Infections screening: RPR-test for Treponema Pallidum, HBsAg, anti-HCV and anti-HIV-antibodies negative, tuberculin skin test - all negative

Autoimmune screening: CRP $18 \mathrm{mg} / \mathrm{L}$; RF, anti-DNA antibodies, anticardiolipin antibodies, pANCA, cANCA, IgG, IgA, IgM - all within normal range.

\section{ECG: otherwise normal}

\section{Chest X-Ray: unremarkable}

Abdomen and kidneys ultrasound: hepatosplenomegaly. Kidneys are enlarged: right $132 \times 50$, left $145 \times 63 \mathrm{~mm}$, with hyperechoic parenchyma $22-25 \mathrm{~mm}$. Mild bilateral collecting system dilatation: right pelvis $20 \mathrm{~mm}$, calices $18 \mathrm{~mm}$, left pelvis $15 \mathrm{~mm}$, calices $10 \mathrm{~mm}$. Multiple small kidney stones 5-7 $\mathrm{mm}$ in calices of both kidneys. No visible retroperitoneal lymph nodes.

Thyroid gland and peripheral lymph nodes ultrasound: unremarkable.

Chest CT: ground-glass areas and small nodules in lower lobes of both lungs. Multiple intrathoracic lymph nodes, located in upper mediastinum, around trachea bifurcation and right lung hilum, diameter up to $11 \mathrm{~mm}$ (Figure 5).

\section{Ophthalmological investigation: unremarkable.}

ENT specialist consult: ENT otherwise normal.

Pulmonologist's consult: Generalized sarcoidosis with lung and intrathoracic lymph nodes involvement.
Treatment: Patient was started with oral prednisone $40 \mathrm{mg} / \mathrm{day}$, normal saline infusions continued.

Treatment results and follow-up: Patient's condition dramatically improved within 2 days, in 10 days his serum creatinine lowered to 190 $\mu \mathrm{mol} / \mathrm{L}$, total calcium decreased to $2.44 \mathrm{mmol} / \mathrm{L}$, ionized calcium - to $1.29 \mathrm{mmol} / \mathrm{L}$. Weakness, eye redness and hoarseness resolved, appetite improved, stool and urination normalized. He was discharged and continued prednisone at home. Outpatient consult a month later: no complains, gained 3 kilos of weight, creatinine $162 \mu \mathrm{mol} / \mathrm{L}$, ionized calcium $1.23 \mathrm{mmol} / \mathrm{L}$, urinalysis otherwise normal. Slow tapering of prednisone was recommended. Chest CT 2 months later showed partial resolution of ground-glass changes and lymph nodes reduction.

At the latest outpatient consult in February 2016 (6 months after the diagnosis and start of treatment) patient doing well, asymptomatic, gained 5 kilos of weight, serum creatinine $136 \mu \mathrm{mol} / \mathrm{L}$, total and ionized calcium within normal range, urinalysis unremarkable. His prednisone dose is $10 \mathrm{mg} /$ day.

\section{Discussion}

Middle-aged male patient with systemic manifestations - erythema nodosum, chest pain, low-gradefever, weightloss, constipation, polyuria, dry mouth, hoarseness and eye redness, gradually developing during 18 months, was admitted to urology clinic with elevated serum creatinine level. He was diagnosed with non-obstructive left pelvis stone, which did not explain his impaired kidney function. That was the indication for kidney biopsy, performed simultaneously with percutaneous nephrolithotomy. Kidney biopsy revealed granulomatous interstitial nephritis and prompted nephrologist consult. Given clinical systemic presentation and pathology findings, sarcoidosis was suspected. Urgent blood test for calcium showed total calcium $3.39 \mathrm{mmol} / \mathrm{L}$, which demanded immediate admission and start of normal saline infusions. Chest CT found intrathoracic lymp node enlargement and fibrotic changes in both lungs, compatible to the diagnosis of sarcoidisis. Search of alternative explanation for pulmonary-renal syndrome with pauci-immune granulomatous interstitial nephritis, ruled out ANCAassociated vasculitis, tubulointerstitial nephritis with uveitis, and tuberculosis. Based on combination of kidney interstitial granulomas, intrathoracic lymph nodes enlargement, hypercalcaemia and history of erythema nodosum, patient was diagnosed with sarcoidosis with lung, intrathoracic lymph nodes, and renal involvement, and treated with $40 \mathrm{mg} /$ day of oral prednisone along with continuous normal saline

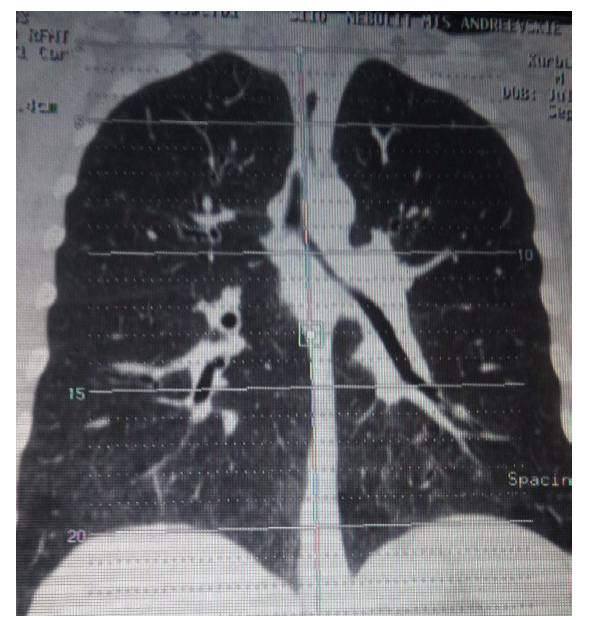

Figure 5. Chest CT. Ground glass and intrathoracic lymph nodes 
infusions. His symptoms resolved dramatically, and normocalcaemia with almost complete recovery of kidney function were achieved, as well as partial resolution of chest CT changes, which supported the diagnosis ex juvantibus.

Retrospective interpretation allow to conclude, that most of signs and symptoms, like polyuria, kidney dysfunction, constipation, weight loss, dry mouth, hoarseness and red eyes were the result of severe hypercalcaemia [8-10]. That is confirmed by substantial recovery of kidney function, which was however incomplete because of prominent interstitial fibrosis, already existing at the time of diagnosis. We suppose that acute kidney injury on the top of chronic kidney disease both occurred due to hypercalcaemia, rather than to granulomatous interstitial nephritis [9,11-13]. Kidney stones (non-obstructive and not influencing kidney function), as well as glycosuria with normal blood glucose, in combination with acidosis, likely resulted from hypercalciuria $[9,11,12]$, which we unfortunately were unable to prove.

Acute kidney injury in sarcoidosis is rare, the most recent data from cohorts of 27 and 13 patients [20,21], confirm that the main cause are a balance anomaly of calcium or a parenchymal involvement. Interestingly, that renal biopsy performed in the second cohort, showed tubulo-interstitial nephritis in all patients, but granuloma only in 6 out of 13 [21].

Coexistence of different types of renal damage in sarcoidosis is very rarely reported in the literature. We could found two small series and 3 case reports. Thus, in 6 patients with renal failure, kidney biopsy demonstrated interstitial nephritis in all cases, combined with nephrocalcinosis in 3, and granulomas in 4 of them [22]. In other series 2 out of 5 patients showed a combination of interstitial nephritis and nephrocalcinosis [7]. In one of the reported case a combination interstitial nephritis and membranous nephropathy was proved in patient with extrapulmonary sarcoidosis [23]. Another case report describes a patient with the history of renal colic, pulmonary-renal sarcoidisis, acute kidney injury and tubulonecrosis plus interstitial granulomas by biopsy [13]. And finally it is reported that in one patient kidney dysfunction was initially attributed to hypercalcaemia, but kidney biopsy, performed because it did not resolved after volume repletion and normalization of serum calcium level, showed nephrocalcinosis and interstitial fibrosis and a single granuloma, not significant in terms of kidney function [12]. However, none of theses cases demonstrate combination of hypercalcaemic acute kidney injury, interstitial granulomatosis and kidney stones, like it was found in our patient.

\section{Conclusion}

Kidney involvement in patients with systemic manifestations demand scrutinous evaluation, including kidney biopsy. Biopsy findings guide further evaluation and may lead to diagnostics of rare conditions, such as sarcoidosis with granulomatous interstitial nephritis. Analysis and careful interpretation of all set of clinical data is mandatory for diagnostics of exceptionally rare conditions like combination of kidney stones, granulomatous interstitial nephritis and hypercalcaemic acute kidney injury.

\section{Acknowledgements}

We have to thank doctors Ruslan Biktimirov, Sergey Borisov and Olga Vinogradova for their impact to diagnostics and treatment of the patient.

\section{Competing interests}

None of authors have any competing interest.

\section{References}

1. Baughman RP, Teirstein AS, Judson MA, Rossman MD, Yeager H Jr, et al. (2001) Clinical characteristics of patients in a case control study of sarcoidosis. Am J RespirCrit Care Med164: 1885-1889. [Crossref]

2. Statement on sarcoidosis (1999) Joint Statement of the American Thoracic Society (ATS), the European Respiratory Society (ERS) and the World Association of Sarcoidosis and Other Granulomatous Disorders (WASOG) adopted by the ATS Board of Directors and by the ERS Executive Committee, February 1999 (1999) Am J RespirCrit Care Med160:736-755.

3. Iannuzzi MC, Rybicki BA, Teirstein AS (2007) Sarcoidosis. N Engl J Med357: 21532165. [Crossref]

4. Mota PC, Morais A, Palmares C, Beltrão M, Melo N, et al. (2012) Diagnostic value of CD103 expression in bronchoalveolar lymphocytes in sarcoidosis. Respir Med106: 1014-1020. [Crossref]

5. Hunninghake GW, Crystal RG (1981) Mechanisms of hypergammaglobulinemia in pulmonary sarcoidosis. Site of increased antibody production and role of $\mathrm{T}$ lymphocytes.J Clin Invest67: 86-92. [Crossref]

6. Facco M, Cabrelle A, Teramo A, Olivieri V, Gnoato M, et al. (2011) Sarcoidosis is a Th1/Th17 multisystem disorder. Thorax 66: 144-150. [Crossref]

7. Le Besnerais M, François A, Leroy F, Janvresse A, Levesque H, et al. (2011) [Renal sarcoidosis: a series of five patients]. Rev Med Interne 32: 3-8. [Crossref]

8. Bergner R, Hoffmann M, Waldherr R, Uppenkamp M (2003) Frequency of kidney disease in chronic sarcoidosis. SarcoidosisVasc Diffuse Lung Dis20: 126-132. [Crossref]

9. Berliner AR, Haas M, Choi MJ (2006) Sarcoidosis: the nephrologist's perspective. Am J Kidney Dis48: 856-870. [Crossref]

10. Rajakariar R, Sharples EJ, Raftery MJ, Sheaff M, Yaqoob MM (2006) Sarcoidtubulointerstitial nephritis: long-term outcome and response to corticosteroid therapy. Kidney Int70: 165-169. [Crossref]

11. Mahévas M, Lescure FX, Boffa JJ, Delastour V, Belenfant X, et al. (2009) Renal sarcoidosis: clinical, laboratory, and histologic presentation and outcome in 47 patients. Medicine (Baltimore) 88: 98-106. [Crossref]

12. Casella FJ, Allon M (1993) The kidney in sarcoidosis. J Am SocNephrol3: 1555-1562. [Crossref]

13. Rema J, Carvalho M, Vaz R, Fonseca M, Sampaio S, et al. (2014) Acute renal failure as a form of presentation of sarcoidosis in a young adult: a case report. $J$ Med Case Rep8: 274. [Crossref]

14. Taylor RG, Fisher C, Hoffbrand BI (1982) Sarcoidosis and membranous glomerulonephritis: a significant association. Br Med J (Clin Res Ed)284: 1297-1298. [Crossref]

15. Göbel U, Kettritz R, Schneider W, Luft F (2001) The protean face of renal sarcoidosis $J$ Am SocNephrol12: 616-623. [Crossref]

16. Iannuzzi MC, Fontana JR (2011) Sarcoidosis: clinical presentation, immunopathogenesis, and therapeutics. JAMA 305: 391-399. [Crossref]

17. Londner C, Zendah I, Freynet O, Carton Z, Dion G, et al. (2011) [Treatment of sarcoidosis]. Rev Med Interne32: 109-113. [Crossref]

18. Hilderson I, Van Laecke S, Wauters A, Donck J (2014) Treatment of renal sarcoidosis: is there a guideline? Overview of the different treatment options. Nephrol Dial Transplant29: 1841-1847. [Crossref]

19. Kearney T, Dang C (2007) Diabetic and endocrine emergencies. Postgrad Med J83: 79-86. [Crossref]

20. Löffler U, Tuleweit A, Waldherr R, Uppenkamp M, Bergner R (2015) Renal Sarcoidosis Epidemiological and Follow-Up Data in a Cohort of 27 Patients. SarcoidosisVasc Diffuse Lung Dis31:306-315. [Crossref]

21. Mahfoudhi M, Gorsane I, Battikh AG, Turki S, Kaaroud H et al (2015) Epidemiological, Clinical and Follow-Up Data in a Series of Thirteen Renal Insufficiencies Complicating Sarcoidosis. Open Journal of Clinical Diagnostics 5:50-53. 
Zakharova EV (2016) Sarcoidosis with combined kidney damage - kidney stones, granulomatous interstitial nephritis and acute kidney injury

22. Bear RA, Handelsman S, Lang A, Cattran D, Wilson D, et al. (1979) Clinical and pathological features of six cases of sarcoidosis presenting with renal failure. Can Med Assoc J121: 1367-1371. [Crossref]
23. Ponce C, Gujral JS (2004) Renal failure and hypercalcemia as initial manifestations of extrapulmonarysarcoidosis. South Med J97: 590-592. [Crossref]

Copyright: (C2016 Zakharova EV. This is an open-access article distributed under the terms of the Creative Commons Attribution License, which permits unrestricted use, distribution, and reproduction in any medium, provided the original author and source are credited. 